\title{
EDITORIAL
}

Bariatric Surgery

\section{Reply: Bariatric surgery and chronic kidney disease: much hope, but proof is still awaited}

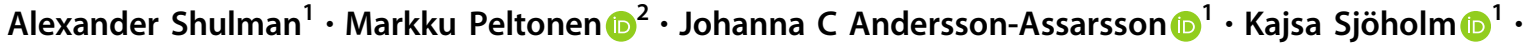 \\ Carel W. le Roux $\mathbb{1}^{3} \cdot$ Per-Arne Svensson $\mathbb{1}^{1,4}$
}

Received: 4 June 2018 / Accepted: 11 June 2018 / Published online: 17 July 2018

(c) Springer Nature Limited 2018

\section{Dear Editor,}

Dr. Arapis et al. has made some important comments on our manuscript.

We agree that failing to account for the presence of competing risk will affect the estimates of cumulative incidence. We have now performed a sensitivity analysis on CKD4/ESRD incidence, taking into account mortality as a competing risk. Using a Kaplan-Meier approach, the 20-year cumulative incidence rates for the CKD4/ESRD event were 1.7 and $3.2 \%$ in the surgery and the control groups, respectively (Figure 2 in the published article). As expected, the corresponding estimates of cumulative incidence of CKD4/ESRD were lower when considering mortality as competing event; 0.7 and $1.4 \%$ in the surgery and the control groups, respectively. The relative risk estimates comparing the surgery group to controls were similar using the Cox regression ( $\mathrm{HR}=0.53,95 \%$ CI $0.32-0.89$ ) and the competing risk regression (adjusted sub-distribution $\mathrm{HR}=0.55,95 \%$ CI 0.33-0.92).

Patients at high risk of developing ESRD (e.g., CKD 2 or CKD 3) are an interesting subgroups when studying the association between bariatric surgery and ESRD. Analyses very similar to the ones suggested by Dr. Arapis et al. are included in the article. The risk-factor treatment interaction

$\triangle$ Per-Arne Svensson

per-arne.svensson@medic.gu.se

1 Department of Molecular and Clinical Medicine, Institute of Medicine, Sahlgrenska Academy, University of Gothenburg, Gothenburg, Sweden

2 Chronic Disease Prevention Unit, National Institute for Health and Welfare, Helsinki, Finland

3 Diabetes Complications Research Centre, Conway Institute, University College Dublin, Dublin, Ireland

4 Institute of Health and Care Sciences, Sahlgrenska Academy, University of Gothenburg, Gothenburg, Sweden analysis (Tables 3 and 4 in the published article) show the study population stratified by eGFR of $91.5 \mathrm{~mL} / \mathrm{min} /$ $1.73 \mathrm{~m}^{2}$. However, since these subgroup analyses did not indicate any difference in relative treatment effects, we did not pursue this any further by performing additional (statistically weaker) subgroup stratifications by CKD stage. In contrast, subgroups defined by baseline albuminuria status or serum insulin level indicate a difference in treatment benefit. Specifically, the results in the macroalbuminuria subgroups, with numbers needed to treat [NNT] between 4 and 8 (compared with NNT between 339 and 616 in patients without albuminuria), are in our opinion clinically very relevant.

We fully agree with Dr. Arapis et al. that a randomized controlled trial is a stronger experimental design to assess causality. Even for high risk patients, there could be "a delay of several years before ESRD." This is of course the major challenge when producing robust evidence for an effect of bariatric surgery on ESRD. Even if ESRD and kidney damage were not predefined endpoints of the SOS study, our article provides the strongest available evidence to date that bariatric surgery is associated with reduced ESRD incidence. We await the results of the BOKID (NCT02612831) and MOMS (NCT01821508) randomized controlled trials with great interest and hope that these results will address some of the knowledge gaps.

\section{Compliance with ethical standards}

Conflict of interest CWIR reports personal fees from Johnson and Johnson, Sanofi Aventis, AstraZeneca, Janssen, Bristol-Myers Squibb, Boehringer-Ingelheim. Other authors declare that they have no conflict of interest. 